\title{
Analytic investigations on the susceptibility of nonlinear analog circuits to substrate noise
}

\author{
C. Widemann, S. Stegemann, W. John, and W. Mathis \\ Institut für theoretische Elektrotechnik, Leibniz Universität Hannover, Appelstraße 9A, 30167 Hannover, Germany \\ Correspondence to: C. Widemann (widemann@tet.uni-hannover.de)
}

\begin{abstract}
This work deals with the conducted susceptibility of nonlinear analog circuits with respect to substrate noise. The substrate coupling mechanism is modeled by a passive three-terminal network that is obtained by means of the finite element method with a subsequently performed model order reduction. Applying this substrate model to the bulk terminal of MOS transistors in integrated analog circuits, it is possible to examine the influence of substrate noise on the circuit's functionality. By means of a block-oriented approach, analytic expressions for the output behavior of the circuits are found. The utilized multi-input Wiener model separates the linear dynamic from the nonlinear static circuit properties. Due to this separation the frequency response of both signals, i.e. input signal and substrate noise, respectively, can be identified, and hence, the frequency range in which the circuit is most susceptible to substrate noise. Since the nonlinear static behavior of each MOS transistor depends on two signals, truncated multivariate Taylor series expansions of the nonlinear elements are performed on the basis of the EKV model description (Enz et al., 1995). The proposed modeling is illustrated by a simple example.
\end{abstract}

\section{Introduction}

The electromagnetic compatibility (EMC) of electronic systems and components became the focus of attention in research and development during the last two decades. The omnipresence of wireless communication systems with high frequencies caused an increasing electromagnetic pollution that endanger the proper functionality of sensitive electronic systems. In this consideration, the role of integrated circuits (IC) which are the core elements of electronic systems can be source and victim of electromagnetic interference (EMI). As source, the ICs radiate or conduct electromagnetic signals that are mainly caused by simultaneous switching noise to

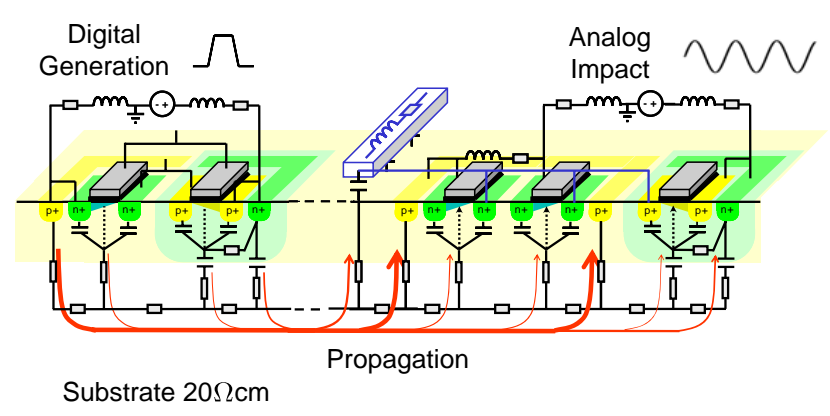

Fig. 1. Substrate coupling in mixed-signal ICs (Bronckers, 2009).

other ICs. In addition to this external EMI, internal chip coupling via the supply network or the shared substrate exists in mixed-signal ICs (cf. Fig. 1). There are several guidelines for IC designers in order to reduce the external and internal coupling, e.g. with decoupling capacitors (Dhia et al., 2006). The impact of substrate noise coupling can be reduced by socalled $p^{+}$guard rings that isolate the active area of NMOS transistors (PMOS are isolated by the $\mathrm{n}$-well) by draining the noise to the off-chip ground (Bronckers, 2009). However, this technique does not attenuate the noise perfectly. Thus, time-varying bulk potentials cause a shift of the DC operating point. Hence, the analog part of mixed-signal ICs is more susceptible to substrate noise than the digital part due to its continuous dynamic range. As a result of interfering signals, intermodulation products in the output spectrum of the nonlinear analog circuits emerge. In order to identify circuit parameters that have direct impact on the emerging intermodulation products, methods for distortion analysis have to be applied. At first, the substrate coupling mechanism is examined in the following section. The resulting model is applied to an exemplary circuit that is evaluated by means of the block-model presented in Sect. 3. 


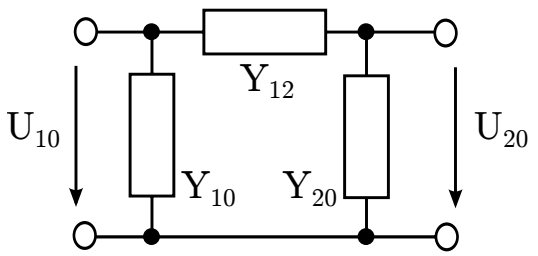

Fig. 2. Three-terminal substrate model (Lin et al., 2009).

\section{Modeling the substrate coupling}

In mixed-signal ICs, the simultaneous switching noise which is the main cause for electromagnetic interference influences the more sensitive analog circuitry. The noise generated by the switching of the digital gates propagates via the shared substrate and impacts the analog active devices. Bronckers (2009) examines the noise coupling mechanism, describes model approaches and introduces possible solutions for the reduction of unwanted effects due to the substrate noise. As depicted in Fig. 1, the substrate possesses resistive and capacitive properties that are modeled by a lumped network. An approximation to the electromagnetic field in the substrate can be found using this lumped network by means of FEM. However, a more practical substrate model is required for further analysis that yield analytic expressions. Thus, exploiting a model order reduction technique leads to a reduced threeterminal substrate model that consists of three delta connected impedances (cf. Fig. 2). Depending on the regarded frequency range, the three impedances are either purely resistive or parallel-connected resistive and capacitive elements (Lin et al., 2009). In the following, this three-terminal model is used in combination with a block model approach for distortion analysis of analog circuits that is reviewed in the next section.

\section{Block oriented EMI analysis}

Due to electromagnetic interference (EMI) the functionality of electronic circuits can be distorted significantly depending on the disturbing signal's frequency and the magnitude. In order to identify circuit parameter that influence the susceptibility to EMI analytical expressions for the unwanted intermodulation products that lie in the signal band are necessary. In general, these analytic expressions can be found by extending the known distortion analysis methods (e.g. Wambacq and Sansen (1998)) with the distortion as additional input signal. The most commonly-used method is a truncated functional series expansion. If nonlinear dynamic systems are regarded, the only possible functional series is the well-known Volterra series. However, even in a multi-tone single input case the so-called Volterra kernels (Weiner and Spina, 1980) are hard to calculate. Hence, for a general multi-input distortion analysis a block-oriented approach is used to model each stage of a multi-stage circuit.

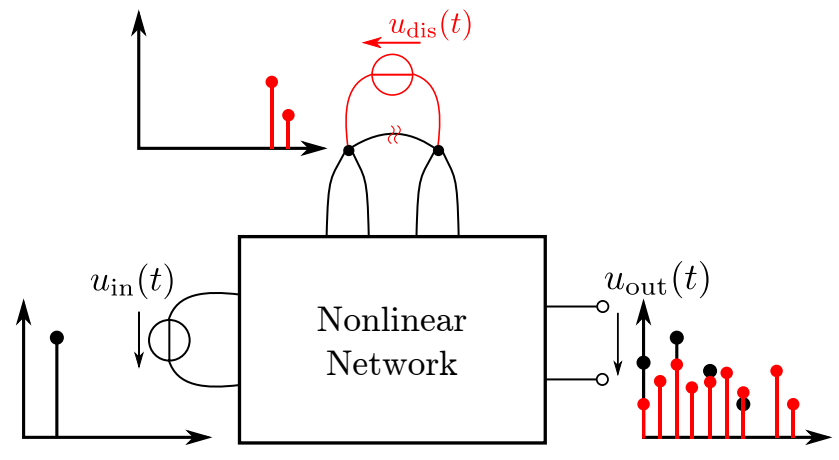

Fig. 3. Modeling the disturbance as independent sources.

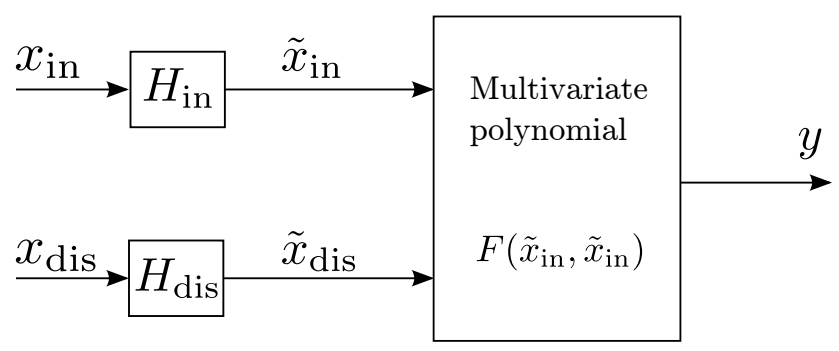

Fig. 4. Block model for distortion analysis (Stegemann et al., 2012).

This approach is based on the classical power series approach for weakly nonlinear single input systems presented e.g. in Weiner and Spina (1980). Moreover, the EMI analysis of nonlinear analog circuits using the known methods for distortion analysis requires that the electromagnetic disturbance can be reduced to a network problem. Thus, the disturbing signals have to be represented by circuit elements, e.g. independent voltage or current sources applied to an arbitrary circuit branch (cf. Fig. 3). As a result, the nominal and the disturbing signal possess their own transfer characteristic with respect to the circuit's output. Hence, a Wiener model approach with multiple input paths is chosen that separates the system into purely linear dynamic blocks and a static nonlinear multi-input block (Stegemann et al., 2012). This block approach is shown in Fig. 4, wherein $x_{\text {in }}$ and $x_{\text {dis }}$ are the generalized input and disturbing signal and $H_{\text {in }}$ and $H_{\text {dis }}$ the corresponding purely linear dynamic transfer characteristic, respectively. The multi-input block $F\left(\tilde{x}_{\text {in }}, \tilde{x}_{\text {dis }}\right)$ represents the static nonlinear behavior of the analog circuit by which intermodulation products between the linearly distorted signals $\tilde{x}_{\text {in }}$ and $\tilde{x}_{\text {dis }}$ emerge in the spectrum of the circuit's output signal $y(t)$. The following sections review the methods to obtain analytic expressions for the different blocks presented in Stegemann et al. (2012) and reveal problems in adapting the method for determining the static nonlinearity $F\left(\tilde{x}_{\text {in }}, \tilde{x}_{\text {dis }}\right)$ for the circumstance of a disturbed substrate. 


\subsection{Linear dynamic block}

The linear dynamic blocks $H_{\text {in }}$ and $H_{\text {dis }}$ are completely described by their frequency domain transfer functions

$H_{\text {in }}(s)=\frac{\tilde{X}_{\text {in }}(s)}{X_{\text {in }}(s)} \quad$ and $\quad H_{\text {dis }}(s)=\frac{\tilde{X}_{\text {dis }}(s)}{X_{\text {dis }}(s)}$.

For the calculation of analytical expressions of these transfer functions the modified nodal analysis (MNA) (Vlach and Singhal, 1983) is applied that results in the frequency domain to the network description

$(s \mathbf{C}+\mathbf{G}) \boldsymbol{Z}(s)=\mathbf{B} \boldsymbol{X}(s)$.

The matrices $\mathbf{C}$ and $\mathbf{G}$ includes the matrix stamps of the dynamic (capacitances and inductances) and the resistive elements, respectively. The vector $\boldsymbol{Z}(s)$ contains the Laplace transforms of the unknown node potentials and currents of the inductances and voltage sources. The right hand side is the product of the matrix $\mathbf{B}$ and the vector $\boldsymbol{X}(s)=$ $\left(X_{\text {in }}(s), X_{\mathrm{dis}}(s)\right)^{T}$ that denotes the frequency description of the signals $x_{\text {in }}(t)$ and $x_{\text {dis }}(t)$. The former maps the independent sources to their corresponding node voltage or branch current (depending on the source type of $x_{\mathrm{in}}(t)$ and $x_{\mathrm{dis}}(t)$ ). The output signals of the linear transfer blocks are obtained by the product of the vector $\boldsymbol{Z}$ and the mapping matrix $\mathbf{L}$ :

$\left[\begin{array}{c}\tilde{X}_{\text {in }}(s) \\ \tilde{X}_{\text {dis }}(s)\end{array}\right]=\mathbf{L Z}(s)$.

Regarding the rows of Eq. (3) including the solution of the linear system of equations (2) separately leads to the both transfer functions

$$
\begin{aligned}
H_{\mathrm{in}}(s) & =\boldsymbol{l}_{1}[s \mathbf{C}+\mathbf{G}]^{-1} \boldsymbol{b}_{1} \\
H_{\mathrm{dis}}(s) & =\boldsymbol{l}_{2}[s \mathbf{C}+\mathbf{G}]^{-1} \boldsymbol{b}_{2},
\end{aligned}
$$

wherein $\mathbf{l}_{1}, \mathbf{l}_{2}$ and $\mathbf{b}_{1}, \mathbf{b}_{2}$ denote the first and second row vector of $\mathbf{L}$ and column vector of $\mathbf{B}$, respectively. Applying these transfer functions to the harmonic Q-tone input signals $x_{\mathrm{in}}, x_{\text {dis }}$ result in (Weiner and Spina, 1980)

$\tilde{x}(t)=\sum_{q=1}^{Q} \hat{x}_{q}\left|H\left(f_{q}\right)\right| \cos \left(2 \pi f_{q} t+\Theta_{q}+\psi_{H}\left(f_{q}\right)\right)$.

Therein, $\hat{x}_{q}$ and $\Theta_{q}$ denote amplitude and phase of the $q^{\text {th }}$ tone of the initial signal $x(t)$ and $\psi_{H}\left(f_{q}\right)$ the phase of the transfer function at the frequency $f_{q}$.

\subsection{Static nonlinear block}

The multivariate polynomial block $F\left(\tilde{x}_{\text {in }}, \tilde{x}_{\text {dis }}\right)$ represents the static nonlinear behavior of the circuit, i.e. without any memory effects of dynamic elements such as capacitances or inductances.
In Stegemann et al. (2012), it was presented how the method of nonlinear current sources (cf.e.g. Schetzen (1985)) for voltage controlled nonlinear circuit elements $i=f_{g}(u)$ can be used for the $N^{\text {th }}$ order approximation of $F\left(\tilde{x}_{\text {in }}, \tilde{x}_{\text {dis }}\right)$. Therein, the dependency $f_{g}$ of each nonlinear element was assumed to be analytic. Hence, the functions were approximated by a truncated power series

$i(t)=f_{g}(u) \approx \sum_{n=1}^{N} a_{n} u^{n}(t)$,

wherein the factors $a_{n}$ denote the nonlinearity coefficients of the nonlinear element of the order $n$ and $u$ the controlling incremental voltage. Applying the iterative method of nonlinear current sources, the MNA leads to systems of equations, each one that corresponds to the order $n$. Thereby, the excitation vector of the $n^{\text {th }}$ order system only depends on the solutions of the systems of orders $m<n$. Hence, an analytic approximation of the order $N$ for the nonlinear output signal $y(t)$ is calculated successively by

$y_{N}(t)=\sum_{i=0}^{N} \sum_{j=0}^{N-i} d_{i j} \tilde{x}_{\mathrm{in}}^{i}(t) \tilde{x}_{\mathrm{dis}}^{j}(t)$.

Rearranging this expression leads to a description with terms that depend either only on $\tilde{x}_{\text {in }}$ or $\tilde{x}_{\text {dis }}$ and mixed terms of both signals

$$
\begin{aligned}
y_{N}(t)= & d_{00}+\sum_{i=1}^{N} d_{i 0} \tilde{x}_{\mathrm{in}}^{i}(t)+\sum_{j=1}^{N} d_{0 j} \tilde{x}_{\mathrm{dis}}^{j}(t)+ \\
& +\sum_{l=2}^{N} \sum_{k=1}^{l-1} d_{(l-k) k} \tilde{x}_{\mathrm{in}}^{l-k}(t) \tilde{x}_{\mathrm{dis}}^{k}(t) .
\end{aligned}
$$

However, in the present problem, the nonlinear elements depend on more than one controlling variable, i.e. $i=$ $f_{g}(u, v, \ldots)$ due to the time-varying bulk potential. Hence, the approach using Eq. (7) is not applicable. Instead, another description is necessary that considers the multi-dimensional dependency such as the multivariate Taylor series (Wambacq and Sansen, 1998) at an operating point $\left(u_{0}, v_{0}, \ldots\right)$ that is given for the two-dimensional case by

$$
\begin{aligned}
i(u, v)= & \sum_{n=0}^{\infty} \frac{1}{n !}\left(\frac{\partial}{\partial u} \Delta u+\frac{\partial}{\partial v} \Delta v\right)^{n} f\left(u_{0}, v_{0}\right) \\
= & f\left(u_{0}, v_{0}\right)+\left.\Delta u \frac{\partial f}{\partial u}\right|_{u_{0}, v_{0}}+\left.\Delta v \frac{\partial f}{\partial v}\right|_{u_{0}, v_{0}}+ \\
& +\frac{1}{2}\left(f_{u u} \Delta u^{2}+2 f_{u v} \Delta u \Delta v+f_{v v} \Delta v^{2}\right)+. .
\end{aligned}
$$

Therein, $\Delta u=u-u_{0}, \Delta v=v-v_{0}$ denote the deviation from the operating point $\left(u_{0}, v_{0}, \ldots\right)$ and $f_{u u}=\left.\frac{\partial^{2} f}{\partial u^{2}}\right|_{u_{0}, v_{0}}$ etc. the partial derivatives. In the following, the influence of substrate noise on a single transistor stage is examined by the use of the proposed model (Fig. 4). 


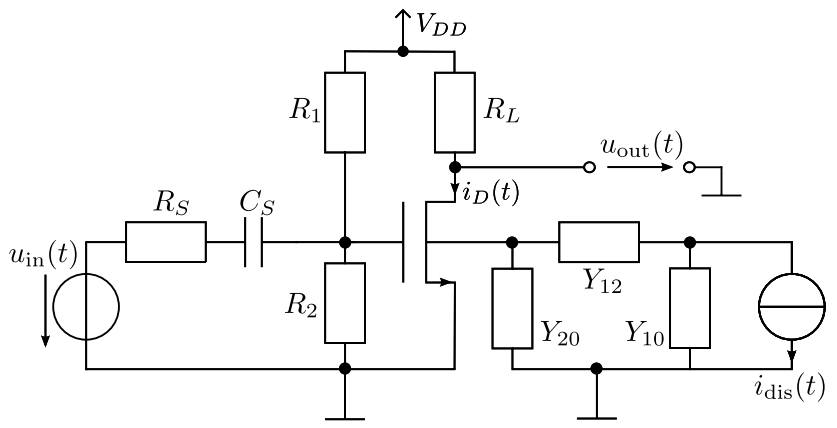

Fig. 5. Common source amplifier stage with gate voltage divider.

\section{Example}

In the following, the proposed method is examined by a simple and hence vivid example. The considered circuit is the common source stage in Fig. 5. The MOS transistor is disturbed by the substrate noise that is modeled as independent current source. The noise couples into the bulk node via the examined three-terminal substrate model (cf. Fig. 2). The gate-source voltage of the MOSFET is set by a gate voltage divider.

\subsection{Characterizing the linear dynamic block}

The characterization of the linear transfer functions requires an equivalent linear small signal model of the circuit (cf. Fig. 6). The transistor is modeled as voltage controlled current source depending on the gate-bulk and source-bulk voltage and capacitances $C_{\mathrm{gb}}$ and $C_{\mathrm{sb}}$. Further elements such as the drain-gate capacitance are neglected. Hence, the individual blocks can be analyzed separately (Weiner and Spina, 1980). The controlling voltages $u_{\mathrm{gb}}$ and $u_{\mathrm{sb}}$ are influenced by both signals, $u_{\text {in }}(t)$ and $i_{\text {dis }}(t)$. The approach shown in Sect. 3.1 leads to an analytic description of

$H_{\text {in }}(s)=\left.\frac{\tilde{U}_{\mathrm{gs}}(s)}{U_{\mathrm{in}}(s)}\right|_{I_{\mathrm{dis}}(s)=0}$,
$H_{\text {dis }}(s)=\left.\frac{\tilde{U}_{\mathrm{sb}}(s)}{I_{\mathrm{dis}}(s)}\right|_{U_{\mathrm{in}}(s)=0}$

in dependency of the network elements. Fig. 7 shows the frequency responses of $H_{\text {in }}(s)$ and $H_{\text {dis }}(s)$ of the analytic expressions. The input transfer function exhibits a bandpass characteristic and the distortion transfer function low-pass characteristic. Due to its impedance character, $H_{\text {dis }}(s)$ has a gain of approximately $20 \mathrm{~dB}$. Thus, the frequency range of largest susceptibility can be identified which is in the present example up to $100 \mathrm{kHz}$ wherein the nominal input signal is attenuated and the distortion signal is not. The input signals of the nonlinear block $\tilde{u}_{\text {in }}$ and $\tilde{u}_{\text {dis }}$, respectively, are determined using (6) assuming only periodic signals that can be expanded into a Fourier series.

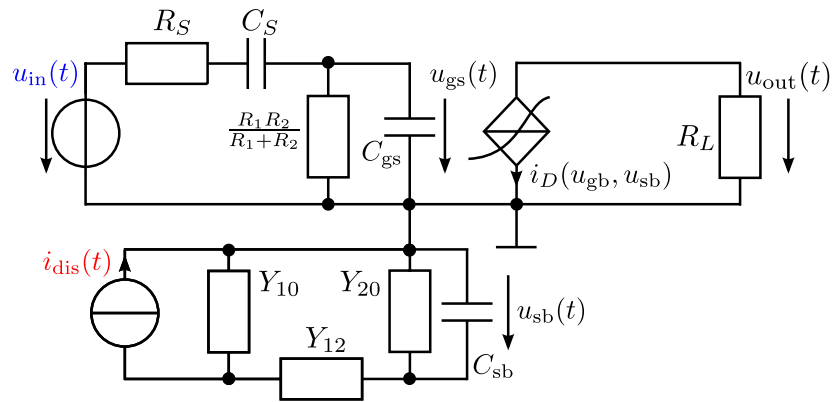

Fig. 6. Small signal model of the disturbed common source stage.

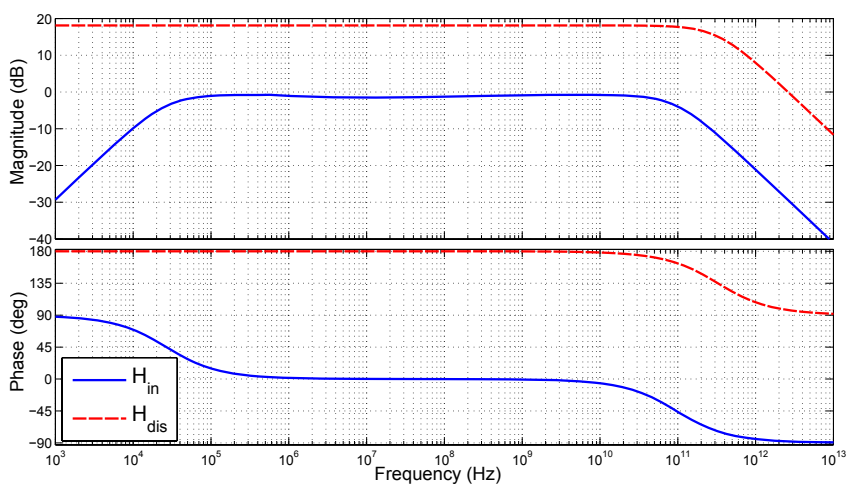

Fig. 7. Frequency response of $H_{\mathrm{in}}$ and $H_{\mathrm{dis}}$.

\subsection{Characterizing the nonlinear static block}

Since the nonlinear block only represents the static behavior of the circuit, the capacitances are neglected and the regarded transconductance $i_{D}=f\left(\tilde{u}_{\mathrm{in}}, \tilde{u}_{\mathrm{dis}}\right)$ is controlled by the previously determined signals (cf. Fig. 8). As an analytic approach to the static dependency, the drain current is assumed to be the forward current of the EKV model (Enz et al., 1995)

$I_{D}\left(U_{\mathrm{gb}}, U_{\mathrm{sb}}\right) \approx\left[\ln \left(1+e^{\frac{V_{P}-U_{\mathrm{sb}}}{2 U_{t}}}\right)\right]$.

Here, $U_{t}=\frac{k t}{q}$ denotes the thermal voltage and $V_{P}$ the pinchoff voltage given by

$$
\begin{aligned}
V_{P}= & U_{\mathrm{gb}}-V_{\mathrm{th} 0}-\gamma\left[\sqrt{U_{\mathrm{gb}}-V_{\mathrm{th} 0}+\left(\sqrt{\Psi_{0}}+\frac{\gamma}{2}\right)^{2}}\right. \\
& \left.-\left(\sqrt{\Psi_{0}}+\frac{\gamma}{2}\right)\right] .
\end{aligned}
$$

with the extrapolated threshold voltage $V_{\text {th0 }}$, the body effect factor $\gamma$ and the surface potential for strong inversion at equilibrium $\Psi_{0}$. Transferring the static expression for the drain current (13) to the multivariate Taylor series (10), an analytic expression for the time-varying drain current in dependency of the intermediate signals with $u_{\mathrm{gb}}=\tilde{u}_{\text {in }}+\tilde{u}_{\text {dis }}$ and 


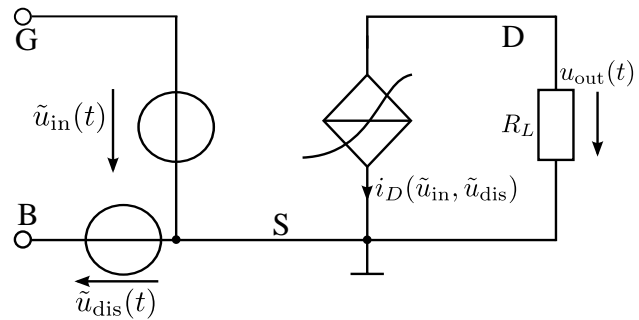

Fig. 8. Two-dimensional voltage controlled current source.

$u_{\mathrm{sb}}=\tilde{u}_{\text {dis }}$ can be determined

$i_{D_{N}}(t)=f\left(\tilde{u}_{\mathrm{in}}, \tilde{u}_{\mathrm{dis}}\right)$

$=\sum_{m=1}^{N} \sum_{n=0}^{m}\left(\left.\frac{\tilde{u}_{\text {in }}^{n}(t)}{n !} \frac{\tilde{u}_{\mathrm{dis}}^{m-n}}{(m-n) !} \frac{\partial^{m} i_{D}\left(\tilde{u}_{\mathrm{in}}, \tilde{u}_{\mathrm{dis}}\right)}{\partial \tilde{u}_{\mathrm{in}}^{n} \partial \tilde{u}_{\mathrm{dis}}^{m-n}}\right|_{\mathrm{OP}}\right)$.

As a result, a dependency of the output current on the input signals and the circuit parameters that influence the Taylor coefficients and the linear transfer functions is found. Comparative simulations are accomplished using these expressions whereby for the use of simplicity the signals $u_{\text {in }}(t)$ and $i_{\text {dis }}(t)$ are single tones, respectively. The frequencies are set to $f_{\text {in }}=10 \mathrm{kHz}$ and $f_{\text {dis }}=3 \mathrm{kHz}$ since in this frequency range the input signal $u_{\text {in }}(t)$ is attenuated noticeably. For validation, a comparison between a harmonic balance simulation in Spectre and the analytic expression found by the block approach is performed, both for amplitudes of $\hat{u}_{\text {in }}=100 \mathrm{mV}$ and $\hat{i}_{\text {dis }}=25 \mathrm{~mA}$. The resulting output spectra for terms up to the third order are shown in Fig. 9 and reveal a very good match for the block model approach.

\section{Conclusions}

In this contribution, an approach for the susceptibility analysis of nonlinear analog circuits due to substrate noise has been presented. The coupling mechanism of digitally generated noise has been modeled as a three-terminal network excited by an independent current source. This model has been used in combination with a multi-input Wiener model proposed in Stegemann et al. (2012) in order to get an analytic approximation of the input-output characteristic of the disturbed system. Hence, circuit designers are equipped with equations for the estimation of most crucially influencing parameter. In addition, the frequency range in which the circuits are the most susceptible to distortion signals can be found. The block-oriented approach is based on classic methods for the distortion analysis, e.g. Wambacq and Sansen (1998). It has been discussed how the blocks can be determined in order to examine the influence on a single transistor. Nevertheless, it has been noted that the MNA based method presented in Stegemann et al. (2012) cannot be adopted for complex circuits due to the multivariate power series approach for each nonlinear element. Thus, future work will deal with this

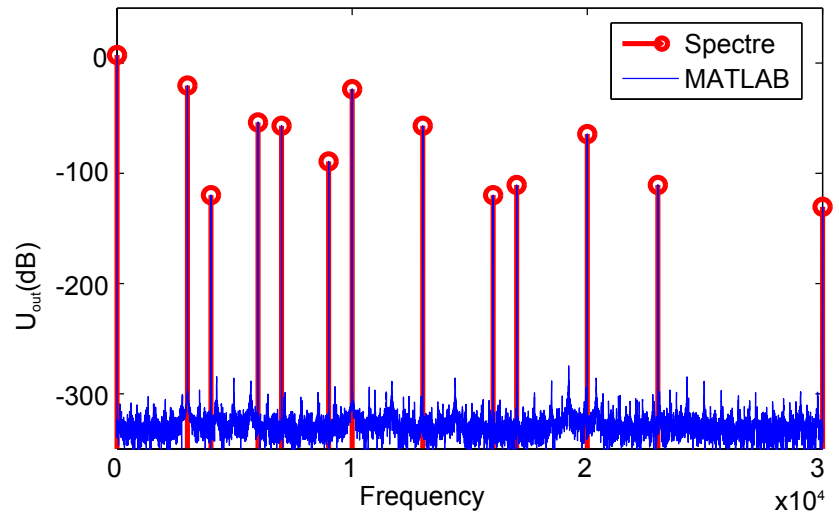

Fig. 9. Spectrum of the output voltage $u_{\text {out }}$.

drawback. One possible approach could be the use of onedimensional power series expansions with the consideration of time-variant operating points due to the substrate noise.

Acknowledgements. This contribution was developed within the scope of the project MAS which is funded by the BMBF (Bundesministerium für Bildung und Forschung) under the project number $16 S V 4059$. The responsibility for this publication is held by the authors only.

\section{References}

Bronckers, S.: Substrate Noise Coupling in Analog/RF Systems, Ph.D. thesis, Vrije Universiteit Brussels, 2009.

Dhia, S. B., Ramdani, M., and Sicard, E. (Eds): Electromagnetic compatibility of integrated circuits techniques for low emission and susceptibility, Springer, New York, 2006.

Enz, C. C., Krummenacher, F., and Vittoz, E. A.: An analytical MOS transistor model valid in all regions of operation and dedicated to low-voltage and low-current applications, Analog Integr. Circuits Signal Process., 8, 83-114, http://dx.doi.org/10.1007/ BF01239381, 1995.

Lin, L., Xiong, J., and Mathis, W.: Modellierung und Simulation des Substrat-Rauschens in integrierten RF CMOS-Schaltungen, Adv. Radio Sci., 7, 163-168, doi:10.5194/ars-7-163-2009,2009.

Schetzen, M.: Multilinear theory of nonlinear networks, Journal of the Franklin Institute, 320, 221-247, 1985.

Stegemann, S., John, W., C.Widemann, and Mathis, W.: A BlockOriented Approach to EMI-induced Distortion Analysis for Nonlinear Analog Circuits, in: EMC EUROPE 2012, Roma, 2012.

Vlach, J. and Singhal, K.: Computer Methods for Circuit Analysis and Design, Van Nostrand Reinhold, 1983.

Wambacq, P. and Sansen, W.: Distortion Analysis of Analog Integrated Circuits, Kluwer Academic Publishers, 1998.

Weiner, D. D. and Spina, J. F.: Sinusoidal Analysis and Modeling of weakly nonlinear Circuits, Van Nostrand Reinhold, 1980. 